Research Paper

\title{
Inhibition of USPI induces apoptosis via IDI/AKT pathway in B-cell acute lymphoblastic leukemia cells
}

\author{
Xingyi Kuang1,2,3,4, Jie Xiong1,2,3, Tingting Lu33, Weili Wang33, Zhaoyuan Zhang33, Jishi Wang1,2,3凶 \\ 1. Department of Hematology, The Affiliated Hospital of Guizhou Medical University, Guiyang 550004, P.R. China. \\ 2. Guizhou Province Hematopoietic Stem Cell Transplantation Center, The Affiliated Hospital of Guizhou Medical University, Guiyang 550004, P.R. China. \\ 3. Key Laboratory of Hematological Disease Diagnostic \& Treat Centre of Guizhou Province, Guiyang 550004, P.R. China. \\ 4. School of Basic Medical Sciences, Guizhou Medical University, Guiyang 550025, P.R. China. \\ $\square$ Corresponding author: Jishi Wang, Department of Hematology, The Affiliated Hospital of Guizhou Medical University, No. 28 Guiyi Street, Yunyan District, \\ Guiyang 550004, P.R. China. Fax: +86-0851-6757898, E-mail: wangjishi9646@163.com.
}

(c) The author(s). This is an open access article distributed under the terms of the Creative Commons Attribution License (https://creativecommons.org/licenses/by/4.0/). See http://ivyspring.com/terms for full terms and conditions.

Received: 2020.04.29; Accepted: 2020.10.08; Published: 2021.01.01

\begin{abstract}
Deubiquitylating enzyme ubiquitin-specific protease I (USPI) has been reported to be aberrantly overexpressed in cancers, and it plays a critical role in regulating various cellular processes, such as cell proliferation, apoptosis, and cell differentiation. However, the role of USPI in B-cell acute lymphoblastic leukemia (B-ALL) remains largely undefined. USPI expression in 30 newly diagnosed B-ALL patients was detected by real-time PCR and western blot. We found that USPI was generally upregulated in the bone marrow cells derived from B-ALL patients. Knockdown of USPI by siRNA decreased B-ALL cell growth and induced apoptosis. Similarly, pharmacological inhibition of USPI by SJB3-0I9A significantly repressed cell proliferation and triggered B-ALL cell apoptosis. Finally, we found that inhibition of USPI downregulated the expression of IDI and P-AKT, and upregulated IDI expression could reverse the suppressive effects of USPI inhibitor in B-ALL cells. Taken together, these results demonstrate that USP I promote B-ALL progression at least partially via the IDI/AKT signaling pathway, and USPI inhibitors might be promising therapeutic application for B-ALL.
\end{abstract}

Key words: B-cell acute lymphoblastic leukemia, USP1, SJB3-019A, ID1, PI3K/AKT pathway, apoptosis

\section{Introduction}

B-cell acute lymphoblastic leukemia (B-ALL), the most common type of malignancy in children and young adults, is a genetically heterogeneous disease that derives from $B$ cell progenitors [1]. In spite of the great advances in the treatment of B-ALL, tumor relapse in B-ALL is one of the leading causes of cancer-related death in childhood [2]. Adults with B-ALL experience even higher relapse rates, with long-term event-free survival less than $50 \%$ [3]. Until now, the therapy of refractory/recurrent B-ALL remains particularly challenging. Therefore, it is necessary to explore novel therapeutic approaches to improve the outcome of B-ALL patients.

Ubiquitination is a very common posttranslational modification, which is reversible. Deubiquitinases (DUBs) are responsible for removing ubiquitin moieties from ubiquitinated substrate proteins, thus reducing their proteasomal degradation and maintaining the balance between ubiquitination and deubiquitination [4]. In addition, the aberrant expression or function of DUBs generally leads to the pathogenesis and progression of a series of cancers [5-7]. Ubiquitin-specific protease 1 (USP1) is one of the best-characterized members of DUBs, playing a critical role in regulating DNA repair processes and cell differentiation [8]. More importantly, the aberrant expression of USP1 is closely associated with the tumorigenesis and progression of multiple cancers [9-11]. Cumulative evidences have shown the abnormal overexpression of USP1 in malignant tumors $[6,12,13]$. Notably, downregulation of USP1 could inhibit cell proliferation and promote apoptosis in a variety of solid tumors $[9,11,12]$. In hematological malignances, downregulation of USP1 inhibited the proliferation of multiple myeloma (MM) and myeloid leukemia cells and induced cell apoptosis [13,14]. 
However, to date, the role of USP1 in B-ALL remains unclarified.

In this study, we examined the expression of USP1 in bone marrow mononuclear cells (BM-MNCs) of 30 B-ALL patients and 18 healthy donors. Afterwards, siRNA and small molecular inhibitor were used to downregulate USP1 expression, aiming to further investigate the functional significance of USP1 in B-ALL-derived cell lines. Finally, we explored the mechanisms of the biological effects of USP1 on B-ALL cells.

\section{Materials and methods}

\section{Reagents and antibodies}

USP1 inhibitor SJB3-019A and proteasome inhibitor MG132 were purchased from MedChem Expression (New Jersey, USA). Antibodies against USP1, AKT, p-AKT were obtained from Cell Signaling Technology (MA, USA). Antibodies specific for $\beta$-actin were purchased from MDL biotech (Beijing, China). Monoclonal antibody against ID1 was bought from Santa Cruz (Heidelberg, Germany).

\section{Patient samples}

Primary human B-ALL patient bone marrow samples were collected from The Affiliated Hospital of Guizhou Medical University. Age-matched healthy donors at Hematopoietic Stem Cell Transplantation Center of The Affiliated Hospital of Guizhou Medical University were also included. Mononuclear cells were separated from bone marrow by Ficoll gradient centrifugation. This study was approved by the institutional review board of The Affiliated Hospital of Guizhou Medical University and all participants offered informed consent according to the Declaration of Helsinki. Patients' characteristics are provided in Table 1.

\section{Cell culture}

Human B-ALL cell lines CCRF-SB, Sup-B15 and KOPN-8 were cultured in complete medium containing $10 \%$ fetal bovine serum (Tianhang Biotechnology, Zhejiang, China) and antibiotics (Invitrogen, Carlsbad, USA). CCRF-SB, Sup-B15 were cryopreserved in Key Laboratory of Hematological Disease Diagnostic \& Treat Centre of Guizhou Province. KOPN-8 cell line was obtained from Beijing Jingzhun Medical Technology Co., Ltd.

\section{Cell viability assay}

The cell viability was assessed using the cell counting kit-8 (CCK-8) test. In brief, B-ALL cells were inoculated into 96-well plates with a density of $5 \times 10^{3}$ cells/well. Then the cells were cultured overnight and treated with different approaches. Afterwards, $10 \mu \mathrm{L}$ of CCK-8 solution (Dojindo, Kumamoto, Japan) was added to each well and incubated at $37^{\circ} \mathrm{C}$ for $2 \mathrm{~h}$, and absorbance values at $450 \mathrm{~nm}$ were measured via spectrophotometer (Molecular Devices, Sunnyvale, California, USA). The cell survival rate (SR) was calculated according to the following formula: SR (\%) $=(\mathrm{OD}$ Treatment /OD Control $) \times 100 \%$. The GraphPad Prism 8 software (GraphPad Software, San Diego, USA) was used to measure the IC50 value.

Table I. Patients' characteristics

\begin{tabular}{ll}
\hline & Descriptive statistics \\
\hline Total patients & 30 \\
Median age, years (minimum-maximum) & $23(1-48)$ \\
Age & \\
$<10$ & $6(20 \%)$ \\
$10-40$ & $19(63 \%)$ \\
$>40$ & $5(17 \%)$ \\
Gender & $11(37 \%)$ \\
Female & $19(63 \%)$ \\
Male & \\
White blood cells count $\mathbf{( \times 1 0} / \mathbf{L})$ & $15(50 \%)$ \\
$<10$ & $9(30 \%)$ \\
$10-99$ & $6(20 \%)$ \\
$\geq 100$ & $8(27 \%)$ \\
Cytogenetics & $1(3 \%)$ \\
Ph (+) & $21(70 \%)$ \\
TEL-AML1 (+) & \\
Normal & $5(17 \%)$ \\
Immunophenotype & $7(23 \%)$ \\
Pro-B-ALL & $18(60 \%)$ \\
Pre-B-ALL &
\end{tabular}

\section{Apoptosis assay}

Apoptosis was determined by Annexin V-FITC and propidium iodide (PI) double staining according to the manufacturer's instructions (7Sea Biotech, Shanghai, China). In brief, B-ALL cells of each group were harvested and washed with PBS once. Cells were then resuspended with $300 \mu \mathrm{l}$ of binding buffer, and incubated with $3.5 \mu \mathrm{L}$ of Annexin V-FITC at room temperature for $15 \mathrm{~min}$ and then with $5 \mu \mathrm{L}$ of PI at 4 ${ }^{\circ} \mathrm{C}$ for $5 \mathrm{~min}$. Flow cytometry (BD Biosciences, San Jose, CA, USA) was performed to assess cell apoptosis. Each experiment was performed in triplicate.

\section{Cell cycle analysis}

Cells were harvested, washed with ice-cold PBS, fixed in $70 \%$ ethanol at $4{ }^{\circ} \mathrm{C}$ for more than 2 hours. Afterwards, the fixed cells were washed with PBS, and incubated with RNase and PI (7Sea Biotech) for 30 min at $37^{\circ} \mathrm{C}$ in dark. The cell cycle distribution was analyzed by flow cytometry (BD Biosciences). 
Quantitative reverse transcriptasepolymerase chain reaction (qRT-PCR)

According to the supplier's recommended protocol, total RNAs were isolated from cells using Trizol reagent (Invitrogen, Carlsbad, CA, USA) and reverse-transcribed by the Revertaid First Strand cDNA Synthesis Kit (Thermo Scientific, Waltham, Massachusetts, USA). The quantitative PCR reaction were performed with a SYBR Green PCR Master Mix (TianGen Biotech, Beijing, China) on the ABI 7500 real-time PCR detection system for $10 \mathrm{~min}$ at $95^{\circ} \mathrm{C}$, followed by 40 cycles at $95^{\circ} \mathrm{C}$ for $15 \mathrm{~s}$ and at $60^{\circ} \mathrm{C}$ for $1 \mathrm{~min}$. The sequences of primers were as follows: USP1 F: 5'-TCATTCAATGGTTCTGGCTTA-3', USP1 R: 5'-GGATTATTTGCG-GTTGTGATG-3'; ID1 F: 5'-AGGGGGCAAGAGGAATTACG-3', ID1 R: 5'-TAG GTGTGCA-GAGAGGAGCG-3'; $\beta$-actin F: 5'-GACAT CCGCAAAGACCTG-3', $\beta$-actin R: 5'-GGAAGG-TGG ACAGCGAG-3'.

\section{Western blot analysis}

Cells were lysed using RIPA buffer (Solarbio Science \& Technology, Beijing, China) supplemented with protease and phosphatase inhibitors (Beyotime, Shanghai, China) for protein extraction. Total proteins were separated on SDS-PAGE gel and transferred to PVDF membranes. Membranes were blotted with primary antibodies for 2 hours at room temperature, membranes were then washed with TBST and incubated in secondary anti-rabbit-HRP (Beyotime) or anti-mouse-HRP antibodies (MDL biotech) for $45 \mathrm{~min}$ at room temperature. All protein bands were detected using the ECL reagent (7Sea Biotech) on the Tanon 4200 automatic chemiluminescence image analysis system (Tanon, Shanghai, China).

\section{Immunofluorescence Staining}

After treatment, B-ALL cells were harvested and centrifuged, then fixed with $4 \%$ paraformaldehyde for 15 min and washed with PBS 3 times. Cell membrane permeabilization was performed with $0.1 \%$ Triton-X 100 (Beyotime) for $30 \mathrm{~min}$. Afterwards, cells were incubated with fresh goat serum (5\%) in following $1 \mathrm{~h}$, cells were probed with specific primary antibody against ID1 at $4{ }^{\circ} \mathrm{C}$ overnight. After washing with PBS 3 times again, cells were incubated with the corresponding fluorescent-labeled secondary antibody (Beyotime). Finally, DAPI (Beyotime) was used to stain the nuclei. Fluorescence images were captured under a fluorescence microscope (Leica DM4000B, Wetzlar, Germany).

\section{Small interfering RNA (siRNA)-mediated gene silencing}

B-ALL cells were seeded at a density of $1 \times 10^{5}$ cells/well into a 6-well plate and cultured before transfection. After 12 hours, CCRF-SB and Sup-B15 cells were transfected with synthesized siRNA specifically targeting human USP1 (USP1-siRNA) (TransheepBio, Shanghai, China) or human ID1 (ID1-siRNA) (Santa Cruz) using Lipo6000 Transfection Reagent (Beyotime) according to the manufacturer's procedures. Meanwhile, a scrambled siRNA served as a negative control (NC-siRNA) (TransheepBio). After 48 hours of transfection, the RNA and protein were extracted and analyzed respectively.

\section{Construction of recombinant lentiviral vectors and transfection}

Recombinant lentivirus-V5-D-TOPO-ID1-EGFP (LV-ID1) and control vector lentivirus-V5-D-TOPOEGFP (LV-control) were constructed. At 30-50\% confluence, typically $24 \mathrm{~h}$ after plating, B-ALL cells were transfected with LV-ID1 and LV-control using 5 $\mu \mathrm{g} / \mathrm{ml}$ polybrene (Genechem, Shanghai, China). After 5 days of infection, B-ALL cells expressing green fluorescent protein (GFP) protein was evaluated using fluorescence microscopy and flow cytometry, and the cells stably overexpressing ID1 were confirmed by real-time PCR and western blot.

\section{Statistical analysis}

All statistical analyses were performed with SPSS software 20.0. Data were presented as mean \pm standard deviation (SD). Student's $t$ test was utilized to derive statistical significance when only two groups were compared. For experiments involving multiple comparisons, we performed one-way ANOVA with the Tukey's test to evaluate differences. $P<0.05$ was considered statistically significant.

\section{Results}

\section{USPI expression in B-ALL patients}

Firstly, real-time PCR was utilized to detect the mRNA expression of USP1 in BM-MNCs from newly diagnosed B-ALL patients and healthy controls. As a result, the expression of USP1 was higher in B-ALL patients compared to that in healthy donors (Figure $1 \mathrm{~A}, P<0.01)$. Western blot analysis also revealed that the protein level of USP1 was higher in B-ALL patients in comparison to that in healthy controls (Figure 1B). These findings indicated a potential role of USP1 in the pathogenesis of B-ALL.

\section{The biological effects of USPI inhibitor SJB3-0I9A on B-ALL cells}

To address the functions of USP1 on B-ALL cells, B-ALL cells were treated with different doses of SJB3-019A, a specific inhibitor of USP1. Consequently, 
CCK-8 assay showed that SJB3-019A suppressed the growth of B-ALL cells (CCRF-SB, Sup-B15 and $\mathrm{KOPN}-8)$ in a dose and time-dependent manner (Figure 2B), and these data demonstrated that Sup-B15 cells were the most sensitive to SJB3-019A (Sup-B15 IC50 $=0.349 \mu \mathrm{M}$, CCRF-SB IC50 $=0.504 \mu \mathrm{M}$ and KOPN-8 IC50 $=0.360 \mu \mathrm{M})$ (Figure 2C). Moreover, SJB3-019A induced apoptosis of B-ALL cells in a dose-dependent manner (Figure 2A). To be specific, the apoptosis rate was $7.06 \%$ and $28.29 \%$ in the $0 \mu \mathrm{M}$ and 0.2 $\mu \mathrm{M}$ SJB3-019A group, respectively, in Sup-B15 cells $(P<0.01)$. While in CCRF-SB cells, the apoptosis rate was $7.14 \%$ in the $0 \mu \mathrm{M}$ SJB3-019A group, but increased to $20.88 \%$ in the $0.2 \mu \mathrm{M}$ group $(P<0.01)$. In KOPN-8 cells, the apoptosis rate was $5.82 \%$ and $27.99 \%$ in the $0 \mu \mathrm{M}$ and $0.2 \mu \mathrm{M}$ SJB3-019A group, respectively $(P<0.01)$.

Previous studies have verified that SJB3-019A could cause G1/G0 cell cycle arrest in MM cells [12]. Therefore, we examined the cell cycle distribution of B-ALL cells after treatment with SJB3-019A. However, cell cycle analysis implied that SJB3-019A induced G2/M phase arrest in B-ALL cells (Figure 3A). After treatment with $0.6 \mu \mathrm{M}$ SJB3-019A, the percentage of cells in the G2/M phase increased from $0.90 \%$ to $12.17 \%$ in Sup-B15 cells $(P<0.01)$, and enhanced from $0.97 \%$ to $12.88 \%$ in CCRF-SB cells $(P<0.01)$.

\section{Silencing USP I with siRNA inhibited cell growth}

Afterwards, siRNA was used to knock down the expression of USP1 in Sup-B15 and CCRF-SB cells. As a result, both mRNA and protein expression of USP1 were significantly inhibited in CCRF-SB and Sup-B15 cells (Figure 4A and 4B). Similar to the effects after SJB3-019A treatment, transfection of USP1-siRNA, but not NC-siRNA, decreased B-ALL cell viability (Figure 4C). In addition, knockdown of USP1 resulted in increased spontaneous apoptosis compared with other two control groups (Figure 4D). After transfection with USP1-siRNA, the percentage of apoptotic cells in Sup-B15 significantly increased from $5.86 \pm 2.17 \%$ to $34.70 \pm 3.22 \%(P<0.01)$. In CCRF-SB cells, the percentage of apoptotic cells was $5.37 \pm$ $2.38 \%$ in the NC-siRNA group, which increased to $27.76 \pm 5.55 \%$ in the USP1-siRNA group $(P<0.01)$. Therefore, the above findings showed a critical role of USP1 in survival of B-ALL cells.

\section{Chemical or genetic inhibition of USP I suppressed ID I/AKT pathway in B-ALL cells}

Inhibitor of DNA binding 1 (ID1) protein is a known target of USP1 [10, 13], and it was found significantly correlated with PI3K/AKT pathway [14]. To further explore whether USP1 regulated the ID1/AKT axis in B-ALL cells, B-ALL cells were transfected with USP1-siRNA. As a result, USP1siRNA transfection caused apparently decreased protein levels of USP1, ID1 and p-AKT in B-ALL cells, whereas the protein level of total AKT was not changed obviously (Figure 5A and 5C). Moreover, the administration of proteasome inhibitor MG-132 could rescue the protein level of ID1 in USP1-siRNA-treated B-ALL cells (Figure 5B), suggesting that USP1-siRNA

A

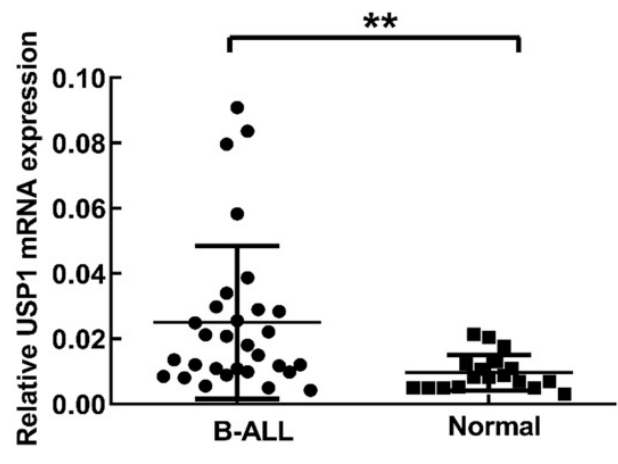

B
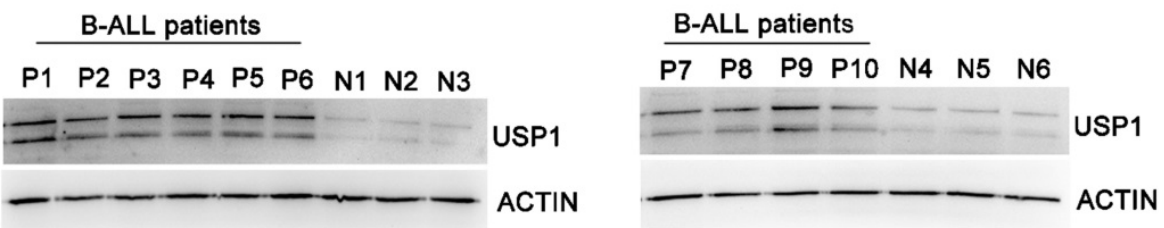

Figure I. Expression level of USPI in B-ALL patients. (A) Detection of mRNA expression level of USPI in BM-MNCs from B-ALL patients and healthy donors using real-time PCR. **, $P<0.0$ I compared with healthy controls. (B) The protein levels of USPI in B-ALL patients were determined by western blot. $\beta$-actin was used as an internal control. 
decreased the protein expression of ID1 through proteasomal degradation. In addition, treatment with SJB3-019A in B-ALL cells attenuated the protein expression of USP1 in a dose-dependent pattern, and concomitantly reduced the protein levels of ID1 and p-AKT (Figure 3B).

A
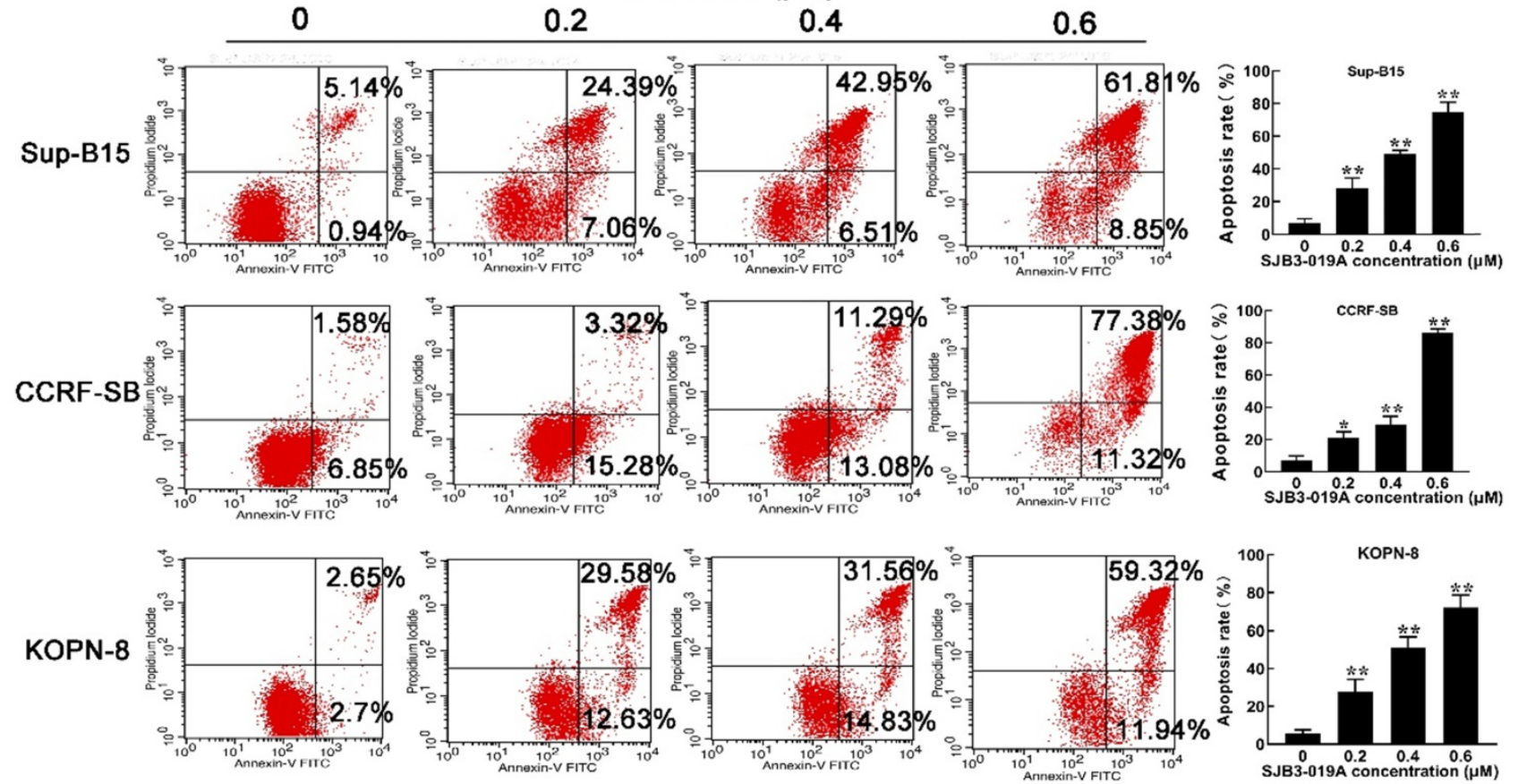

B
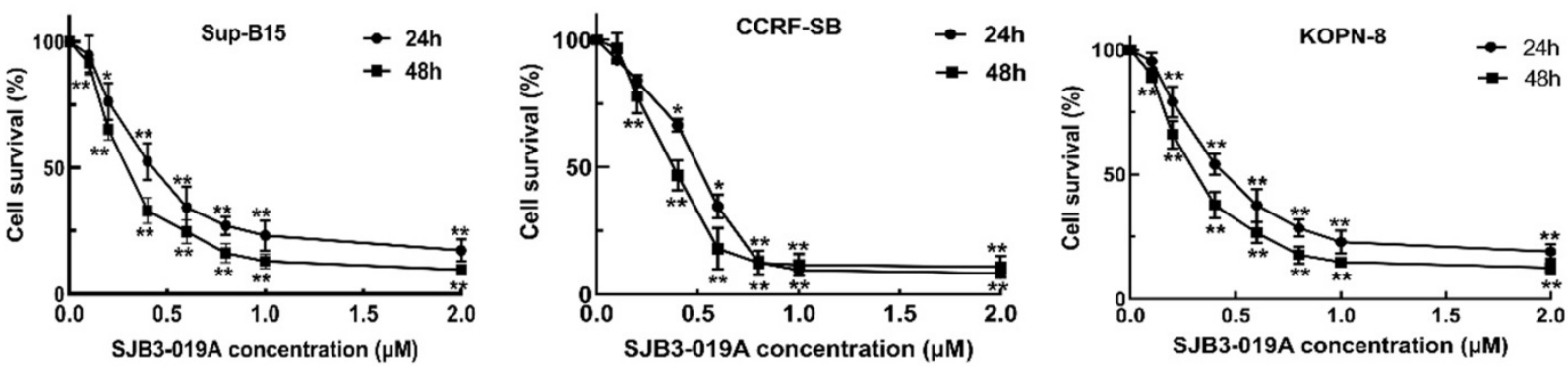

C
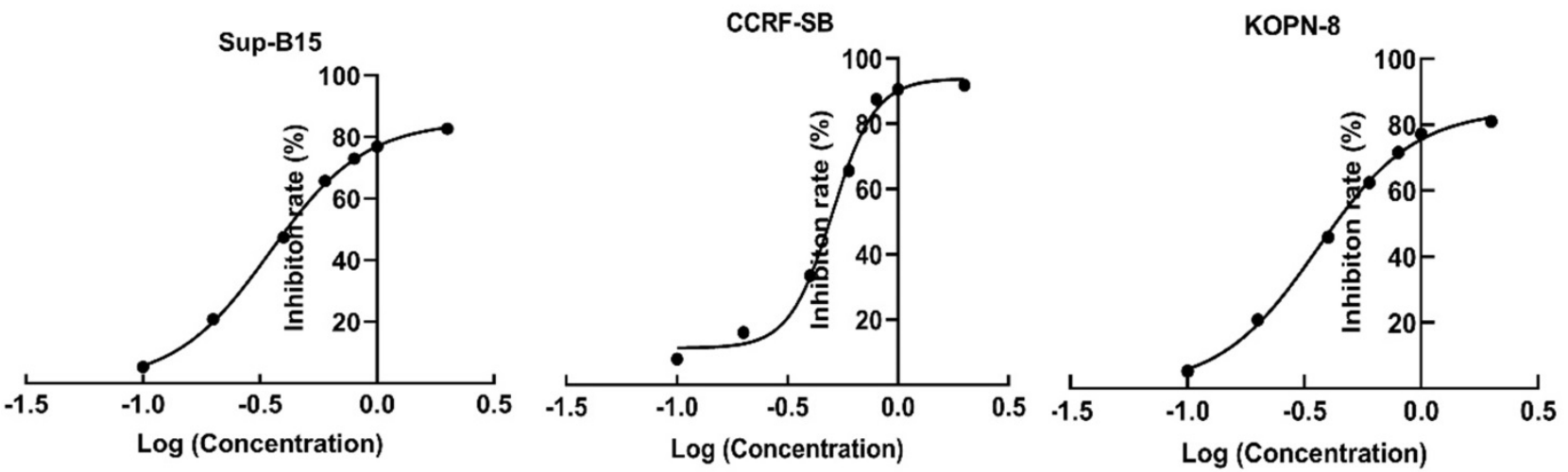

Figure 2. SJB3-0 I9A suppressed cell survival and induced apoptosis in B-ALL cells. (A) Apoptotic rates of CCRF-SB, Sup-BI5 and KOPN-8 were assessed by flow cytometry after treatment with $0,0.2,0.4,0.6 \mu \mathrm{M} \mathrm{SJB} 3-019 \mathrm{~A}$ for $24 \mathrm{~h}$. Data were presented as mean $\pm \mathrm{SD} ; *, P<0.05$ versus $0 \mu \mathrm{M}$, and $* *, P<0.0 \mathrm{I}$ versus $0 \mu \mathrm{M}$ group. (B) B-ALL cells were treated with various concentrations of SJB3-019A for 24 and $48 \mathrm{~h}$, respectively, followed by cell viability evaluation by CCK-8 assay. Data were shown as mean \pm SD; $*, P<0.05$ versus $0 \mu \mathrm{M}$ group; and **, $P<0.01$ versus $0 \mu \mathrm{M}$ group. (C) According to the $\mathrm{OD}_{450}$ values obtained from $C C K-8$ assay, the IC 50 value was analyzed using Graphpad software 8. 
A
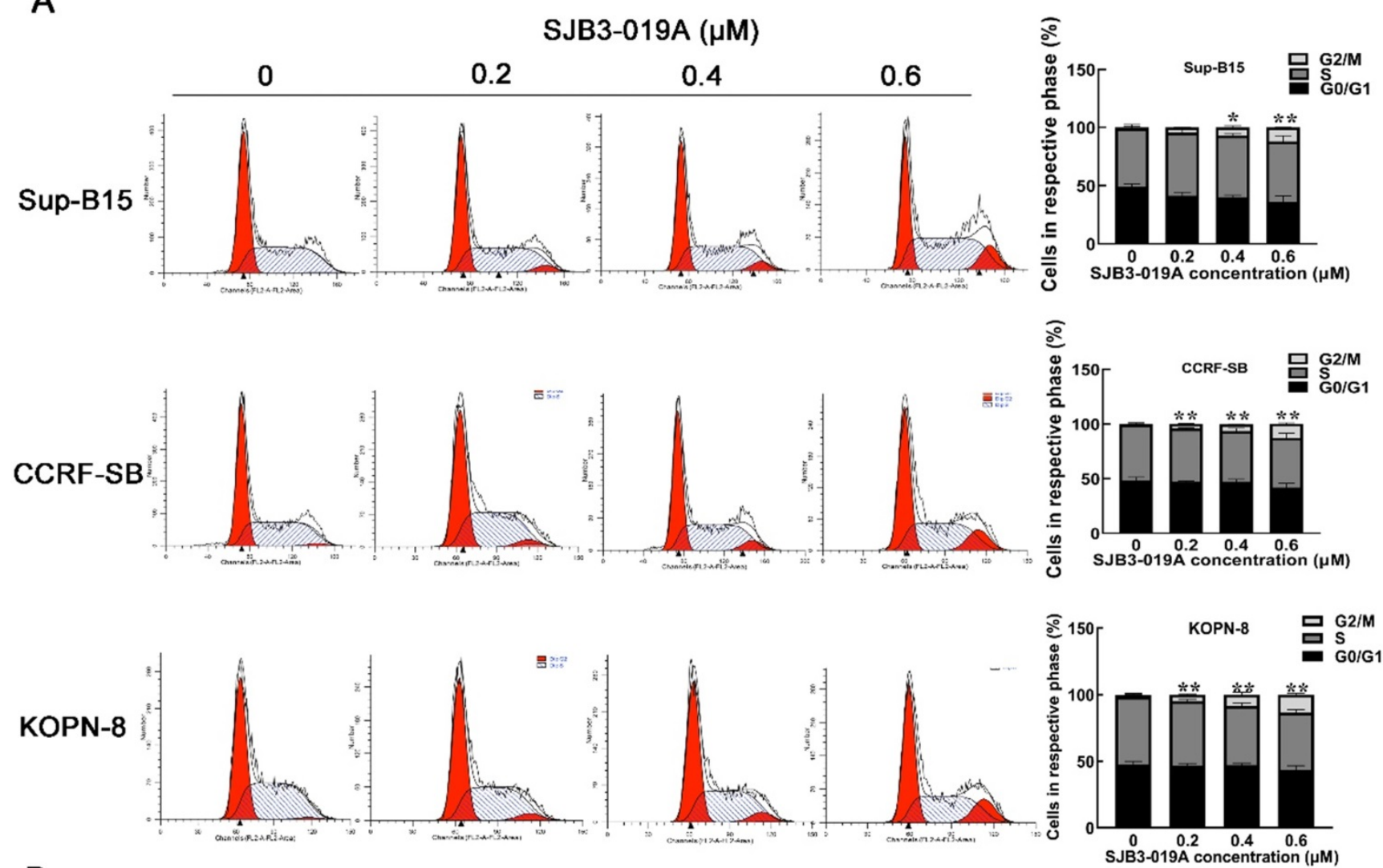

B
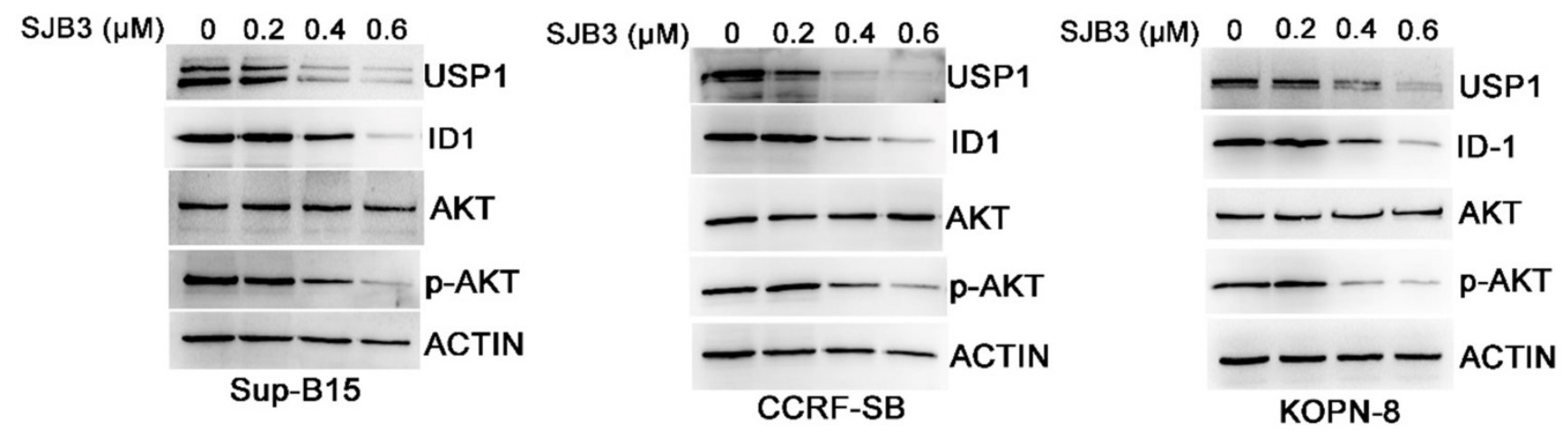

Figure 3. Effects of SJB3-0 I 9A on cell cycle distribution and expression of USP I, IDI and p-AKT. (A) B-ALL cells were incubated with SMI-4a for $24 \mathrm{~h}$, followed by flow cytometry to determine the cell cycle distribution. Data were presented as mean \pm SD; $*, P<0.05$ versus $0 \mu M$ group; and **, $P<0.01$ versus $0 \mu M$ group. All experiments were performed in triplicate. (B) B-ALL cells were treated with SJB3-0I9A for $24 \mathrm{~h}$, followed by detection of the protein expression of USPI, ID I, AKT and p-AKT using western blot analysis. $\beta$-actin was used as a loading control.

\section{SJB3-0 I 9A induced apoptosis in B-ALL cells via IDI/AKT pathway}

Similar to USP1, siRNA-mediated downregulation of ID1 also led to reduced level of p-AKT without effects on total AKT level. Downregulation of ID1 with siRNA caused decreased mRNA and protein expression of ID1, but did not decrease USP1 protein levels (Fig. 6A and 6B), indicating that USP1 functioned as the upstream of ID1 in B-ALL cells. To confirm the role of ID1 in suppressive effects of USP1 in B-ALL cells, lentivirus vector was used to upregulate the expression of ID1 in B-ALL cells. Flow cytometry showed that the percentage of GFPpositive cells was over $80 \%$ after transfection with lentivirus expressing ID1 (LV-ID1) (Supplementary Figure S1A), indicating successful infection. Next, the expression of ID1 was detected using real-time PCR and western blot. As a result, LV-ID1 infection significantly increased both mRNA and protein expression levels of ID1 (Supplementary Figure S1B and S1C). After infection with LV-ID1, the protein level of p-AKT was increased in SJB3-019A-treated cells (Figure 6C). More importantly, upregulated ID1 expression decreased apoptosis induced by SJB3-019A 
in B-ALL cells (Figure 6D). In addition, when B-ALL cells treated with SJB3-019A, upregulating ID1 significantly promoted cell viability (Figure 6E).
Collectively, the present data demonstrated that SJB3-019A induced apoptosis at least partially through ID1-mediated AKT/PI3K pathway.
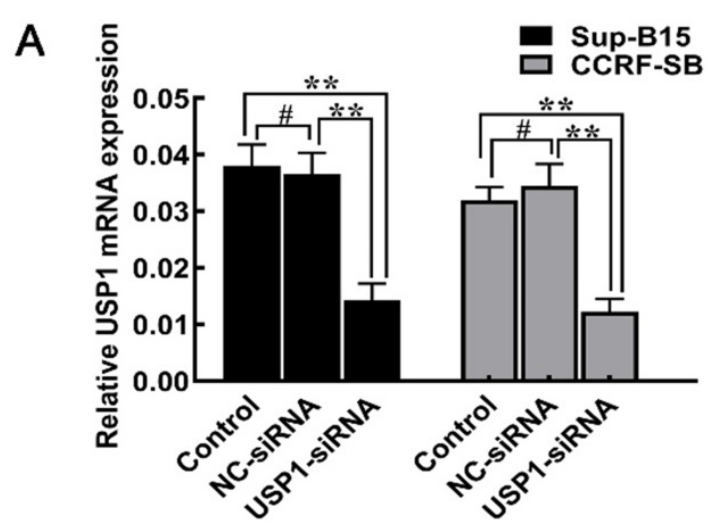

B
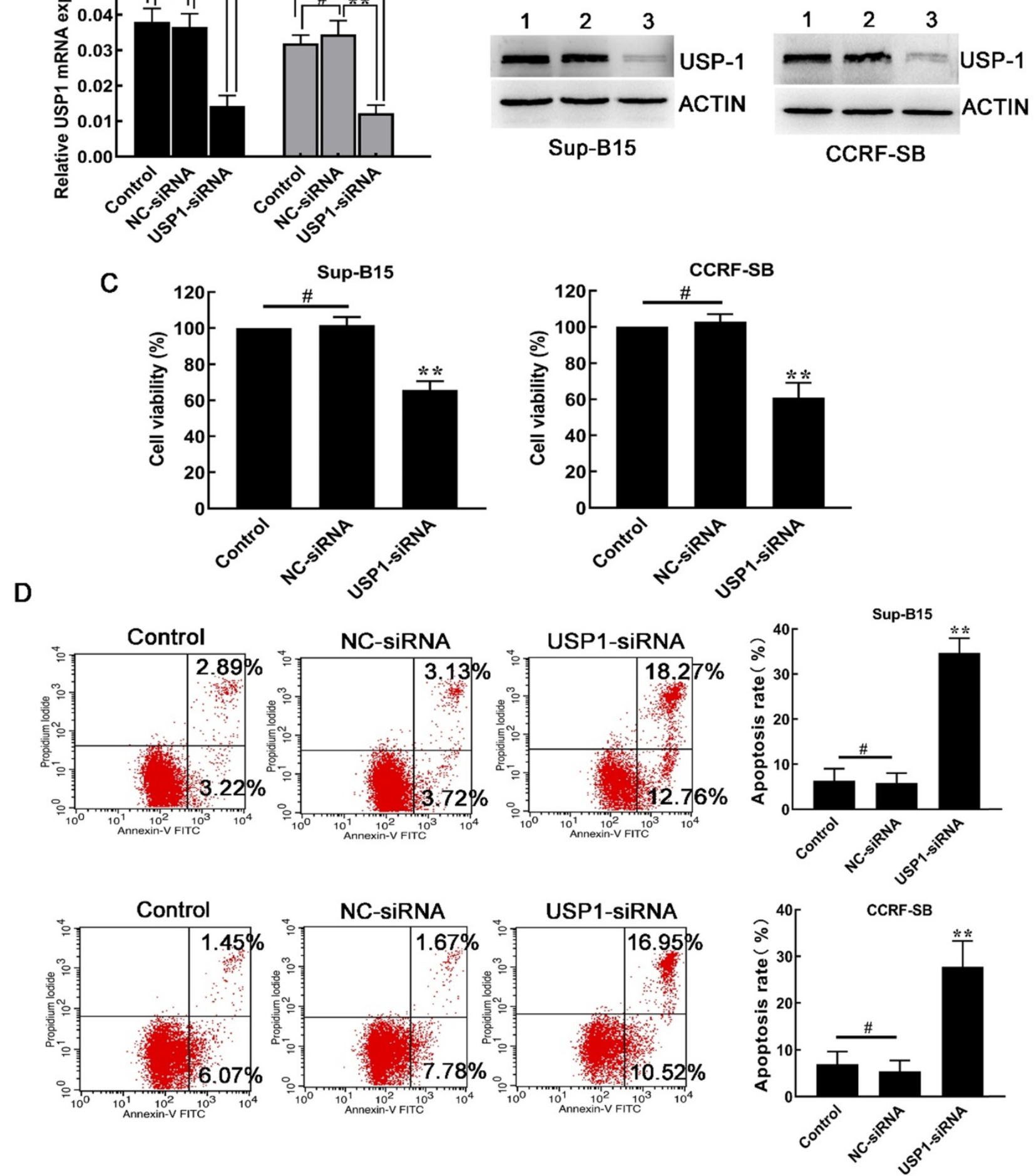

Figure 4. Effects of USPI siRNA in B-ALL cells. (A) After transfection with USPI-siRNA, real-time PCR and was used to quantify the mRNA levels of USPI. **, $P<0.0$ I; $\#, P>0.05$ versus the other two groups. (B) Western blot was used to detect the protein level of USPI. Lane I, Control group; lane 2, NC-siRNA group; lane 3, USPI-siRNA group. (C) Depletion of USPI inhibited the cell growth of CCRF-SB and Sup-BI 5 cells. Data were presented as mean \pm SD; $* *, P<0.0$ I versus other two group; $\#, P>0.05$. (D) The effect of USPI siRNA on apoptosis of CCRF-SB and Sup-BI5 cells was determined by flow cytometry. Data were shown as mean \pm SD; $* *, P<0.0$ I in comparison to the control group; \#, $P>0.05$ 
A

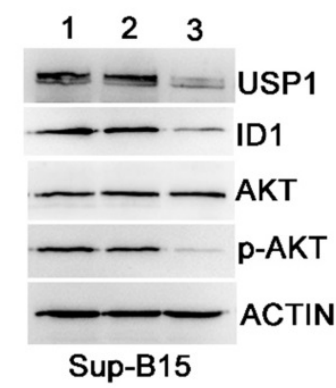

B

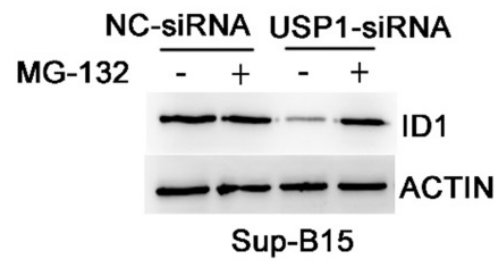

C

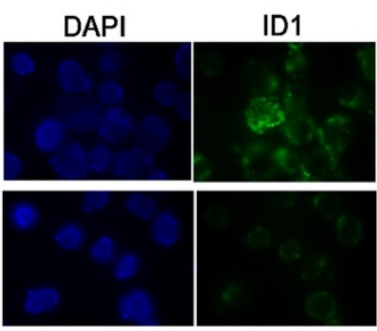

Sup-B15

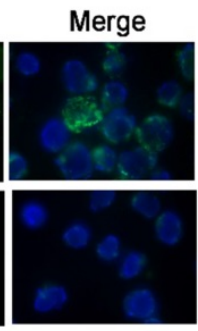$$
\text { (1) }
$$

Figure 5. USPI regulated the expression of IDI/AKT pathway in B-ALL cells. (A) After transfection with USPI-siRNA, the protein expression levels of USPI, IDI, AKT and p-AKT were detected by western blot. Lane I, Control group; lane 2, NC-siRNA group; lane 3, USPI-siRNA group. (B) IDI expression was analyzed by western blot in B-ALL cells with either NC-siRNA or USPI-siRNA in the presence or absence of MG-I32. (C) Immunofluorescence staining of IDI is performed as described in materials and methods. The images shown are under $\times 1000$ magnification. The images are representative of three independent experiments.

\section{Discussion}

B-ALL, the most common type of ALL, is characterized by clonal expansion of developmentally arrested malignant B-cell precursors [2]. Approximately $20 \%$ of B-ALL patients become resistant to chemotherapy during the therapeutic process [15]. Moreover, patients with refractory/ recurrent B-ALL have poor prognosis, without promising therapeutic effects in these patients [3]. Therefore, it is necessary to develop novel therapeutic strategies targeting leukemia-specific molecular determinants for B-ALL.

The overexpression or hyper-activation of DUBs has been widely detected in various cancers, contributing to tumor development and progression [16]. As a member of DUBs, USP1 is overexpressed in various types of cancers and is considered as an oncogene associated with cancer progression, metastasis and drug resistance [6, 11, 12]. Recent studies have demonstrated that USP1 inhibition induced apoptosis and suppressed cell proliferation in acute myeloid leukemia (AML) and MM cells [12, 13]. However, the exact roles of USP1 in B-ALL
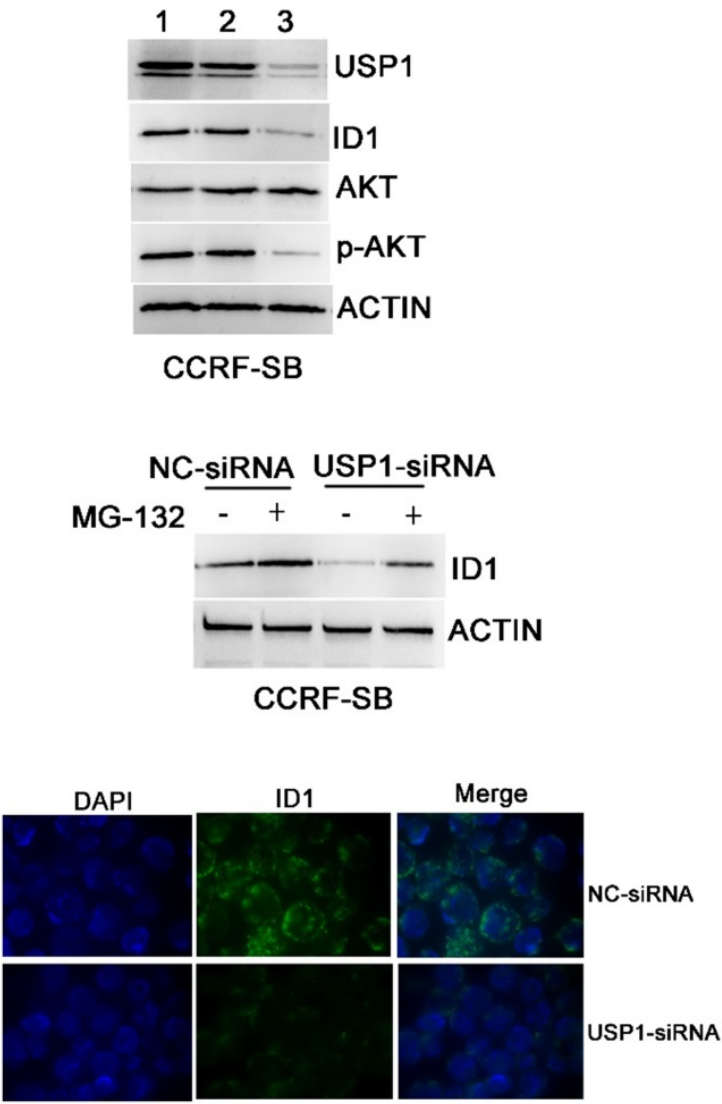
. 
A

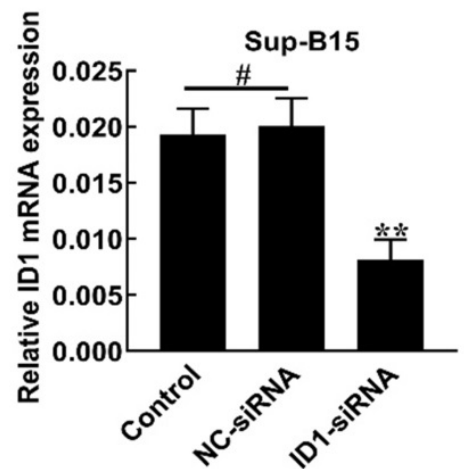

C

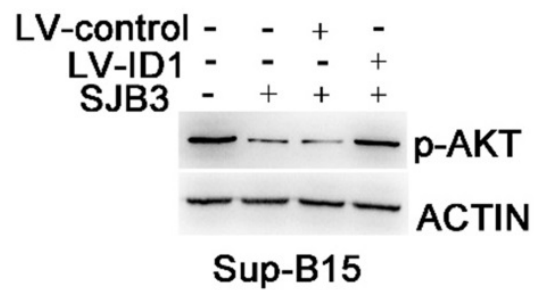

D
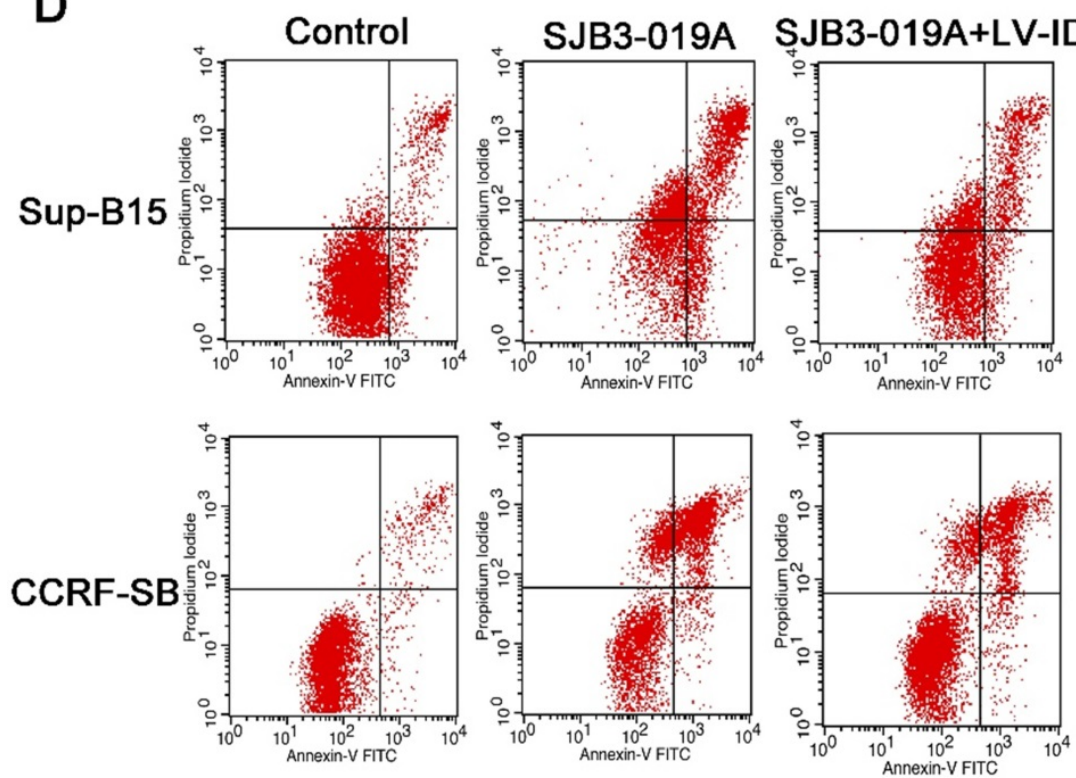

B

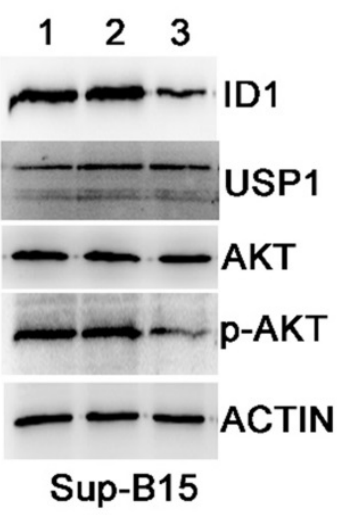

$\begin{array}{lll}1 & 2 & 3\end{array}$

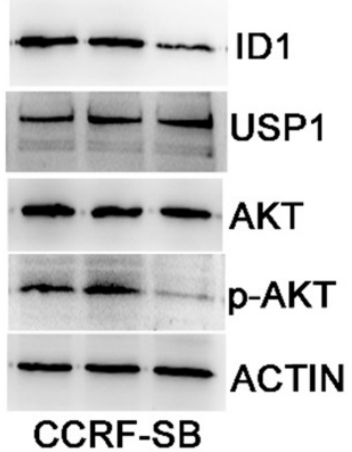

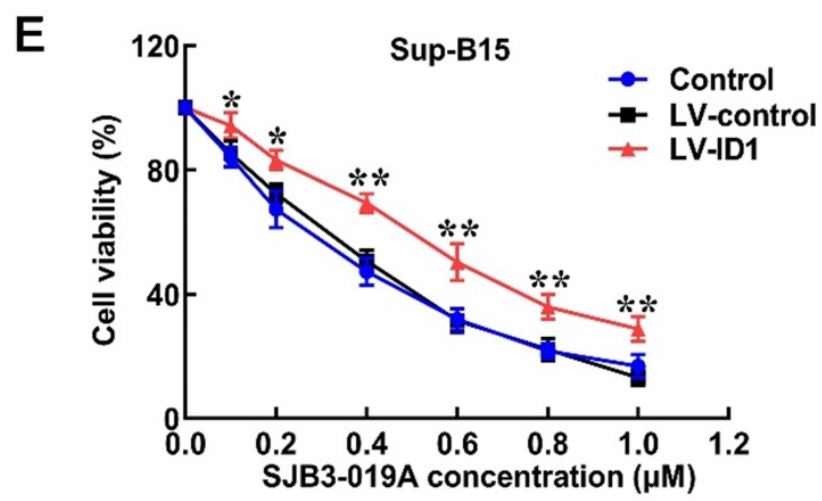

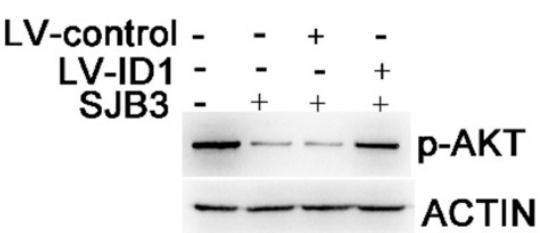

CCRF-SB
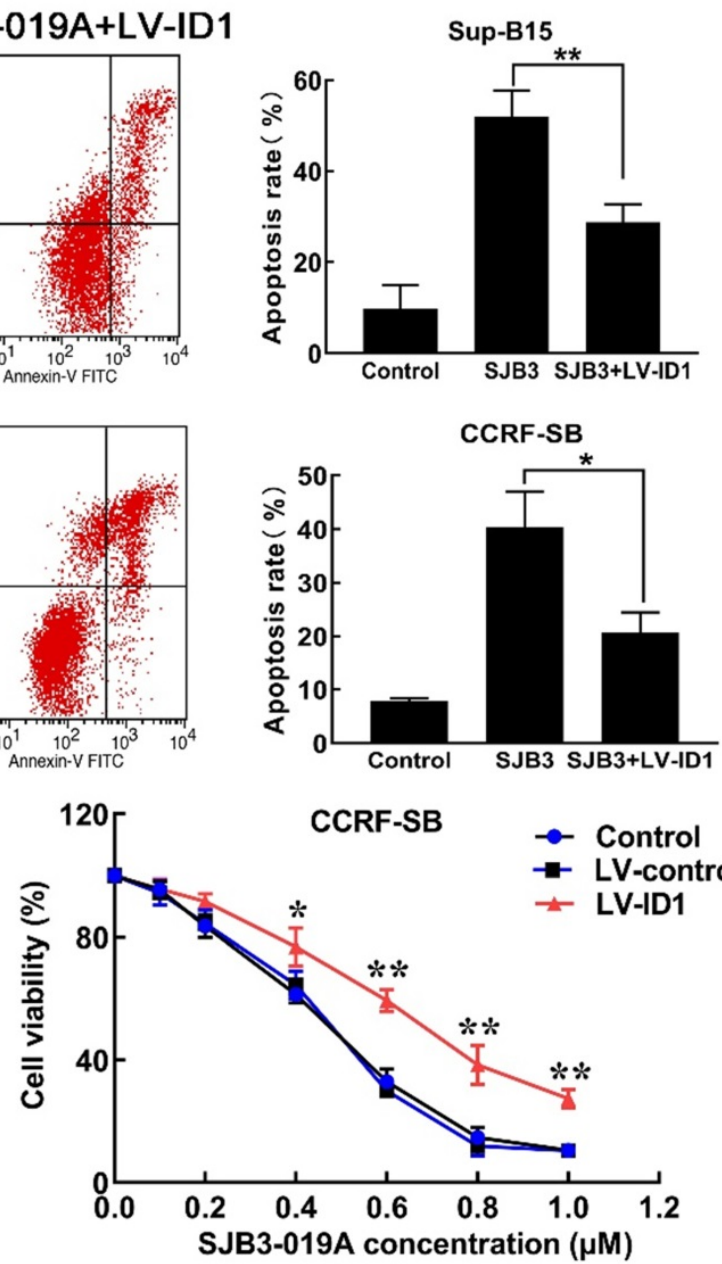

Figure 6. SJB3-0I9A induced B-ALL cell apoptosis partially through IDI-mediated PI3K/AKT pathway. (A) real-time PCR analysis of IDI in B-ALL cells after transfection with either negative control siRNA or siRNA targeting IDI. **, $P<0.0$ I versus other two group; $\#, P>0.05$. (B) Western blot analysis of the protein expression of IDI, USPI, AKT and p-AKT in B-ALL cells. Lane I, Control group; lane 2, NC-siRNA group; lane 3, IDI-siRNA group. (C) B-ALL cells transfected with either LV-control or LV-IDI were incubated with SJB3-019A, followed by detection of the intracellular levels of P-AKT using western blot. (D) Apoptotic rates of CCRF-SB and Sup-BI5 cells after IDI regulation and SJB3-0I9A treatment were examined by flow cytometry. Data were presented as mean \pm SD; *, $P<0.05$, and $* *, P<0.01$. (E) B-ALL cells were infected with LV-control or LV-IDI, and incubated with different concentrations of SJB3-0I9A, followed by cell viability assessment by CCK-8 assay. $*, P<0.05$, **, $P<0.0 \mathrm{I}$ versus the control group and LV-control group. 
ID1 protein is a member of the helix-loop-helix family of transcriptional regulatory proteins. As a known target of USP1 [10, 13], the dysregulation of ID1 has been widely reported in multiple types of human tumors, which is essential for processes such as proliferation, cell migration and stem cell renewal [19-21]. In B-ALL patients, high expression level of ID1 is associated with the poor outcome [22]. Conditional deletion of ID1 prolongs the survival time of AML mice, while ID1 inhibitor obviously inhibits AML cell growth and promotes apoptosis, indicating that ID1 is a critical regulator in leukemogenesis [23]. In our study, both USP1-siRNA and USP1 inhibitor (SJB3-019A) could decrease the protein level of ID1 in B-ALL cells. And the reduction of ID1 protein level caused by USP1-siRNA was mediated by proteasomal degradation, since the proteasome inhibitor, MG-132, rescued the expression of ID1 protein. More importantly, we found that upregulation of ID1 decreased the apoptosis caused by USP1 inhibitor SJB3-019A. These studies indicate that the suppressive role of USP1 in B-ALL cells is partially mediated by ID1.

The PI3K/AKT signaling pathway is involved in a wide range of physiological processes which is often dysregulated in tumorigenesis [24]. In fact, the aberrant activation of PI3K/AKT pathway is very common in various types of cancers including hematological malignancies [25]. Multiple studies have shown that the PI3K/AKT pathway is highly activated in B-ALL cells $[26,27]$, and inhibition of PI3K/AKT pathway leads to apoptotic activation in B-ALL cells [27]. A previous study has proved that inhibition of ID1 significantly decreases p-AKT protein level in AML and osteosarcoma cells [20,23]. To further explore whether USP1 regulates the ID1/ AKT axis in B-ALL cells, the expression of USP1 and ID1 was knocked down in B-ALL cells, respectively. As a result, downregulation of USP1 and ID1 suppressed the PI3K/AKT pathway, as determined by the major reduction in p-AKT protein level. However, ID1 knockdown did not affect USP1, further suggesting USP1 is an upstream of ID1 in B-ALL cells. The present study indicated that USP1 silencing could downregulate the expression of ID1 and suppress the phosphorylation of AKT, suggesting that USP1 regulates PI3K/AKT signaling pathway in B-ALL progression, possibly by regulating ID1 expression. To further determine whether USP1-induced PI3K/AKT activation was mediated by the ID1 expression, B-ALL cells were treated with SJB3-019A and LV-ID1 or LV-control, respectively. Consequently, the upregulation of ID1 increased the protein level of p-AKT in SJB3-019A-treated B-ALL cells. Taken together, these findings demonstrate that inhibition of USP1 suppresses the activation of the PI3K/AKT pathway and promotes B-ALL cell apoptosis by downregulating the expression of ID1.

In conclusion, our current outcomes indicate the overexpression of USP1 in B-ALL patients. Genetic and pharmacologic inhibition of USP1 markedly suppress B-ALL cell growth and induce apoptosis. More importantly, inhibition of USP1 leads to the downregulated expression of ID1, further causing the inactivation of PI3K/AKT pathway. Therefore, USP1 inhibition presents as a novel therapeutic strategy for B-ALL, and future in vivo studies for USP1 inhibition are warranted.

\section{Abbreviations}

B-ALL: B-cell acute lymphoblastic leukemia; USP1: ubiquitin-specific protease 1; ID1: inhibitor of DNA binding 1; AML: acute myeloid leukemia; MM: multiple myeloma; BM-MNCs: bone marrow mononuclear cells; siRNA: small interfering RNA; CCK-8: cell counting kit-8; qRT-PCR: quantitative reverse transcriptase-polymerase chain reaction.

\section{Supplementary Material}

Supplementary figure $\mathrm{S} 1$.

http://www.medsci.org/v18p0245s1.pdf

\section{Acknowledgements}

This study was supported by the National Science Foundation of China (No. 81660616) and Guizhou Provincial Social Research Project Fund (No. Qiankehe [2015]3036+2).

\section{Competing Interests}

The authors have declared that no competing interest exists.

\section{References}

1. Sun W, Malvar J, Sposto R, Verma A, Wilkes JJ, Dennis R, et al. Outcome of children with multiply relapsed B-cell acute lymphoblastic leukemia: a therapeutic advances in childhood leukemia \& lymphoma study. Leukemia. 2018; 32: 2316-25.

2. Velazquez-Avila M, Balandran JC, Ramirez-Ramirez D, Velazquez-Avila M, Sandoval A, Felipe-Lopez A, et al. High cortactin expression in B-cell acute lymphoblastic leukemia is associated with increased transendothelial migration and bone marrow relapse. Leukemia. 2019; 33: 1337-48.

3. Kantarjian H, Jabbour E. Incorporating Immunotherapy Into the Treatment Strategies of B-Cell Adult Acute Lymphoblastic Leukemia: The Role of Blinatumomab and Inotuzumab Ozogamicin. American Society of Clinical Oncology educational book American Society of Clinical Oncology Annual Meeting. 2018; 38: 574-8.

4. Harrigan JA, Jacq X, Martin NM, Jackson SP. Deubiquitylating enzymes and drug discovery: emerging opportunities. Nature reviews Drug discovery. 2018; 17: 57-78.

5. Zhou M, Wang T, Lai H, Zhao X, Yu Q, Zhou J, et al. Targeting of the deubiquitinase USP9X attenuates B-cell acute lymphoblastic leukemia cell survival and overcomes glucocorticoid resistance. Biochemical and biophysical research communications. 2015; 459: 333-9.

6. Ma A, Tang M, Zhang L, Wang B, Yang Z, Liu Y, et al. USP1 inhibition destabilizes KPNA2 and suppresses breast cancer metastasis. Oncogene. 2019; 38: 2405-19.

7. McClurg UL, Robson CN. Deubiquitinating enzymes as oncotargets. Oncotarget. 2015; 6: 9657-68. 
8. Garcia-Santisteban I, Peters GJ, Giovannetti E, Rodriguez JA. USP1 deubiquitinase: cellular functions, regulatory mechanisms and emerging potential as target in cancer therapy. Molecular cancer. 2013; 12: 91.

9. Liu J, Zhu H, Zhong N, Jiang Z, Xu L, Deng Y, et al. Gene silencing of USP1 by lentivirus effectively inhibits proliferation and invasion of human osteosarcoma cells. International journal of oncology. 2016; 49: 2549-57.

10. Williams SA, Maecker HL, French DM, Liu J, Gregg A, Silverstein LB, et al. USP1 deubiquitinates ID proteins to preserve a mesenchymal stem cell program in osteosarcoma. Cell. 2011; 146: 918-30.

11. Lee JK, Chang N, Yoon Y, Yang H, Cho H, Kim E, et al. USP1 targeting impedes GBM growth by inhibiting stem cell maintenance and radioresistance. Neuro-oncology. 2016; 18: 37-47.

12. Das DS, Das A, Ray A, Song Y, Samur MK, Munshi NC, et al. Blockade of Deubiquitylating Enzyme USP1 Inhibits DNA Repair and Triggers Apoptosis in Multiple Myeloma Cells. Clinical cancer research : an official journal of the American Association for Cancer Research. 2017; 23: 4280-9.

13. Mistry H, Hsieh G, Buhrlage SJ, Huang M, Park E, Cuny GD, et al. Small-molecule inhibitors of USP1 target ID1 degradation in leukemic cells. Molecular cancer therapeutics. 2013; 12: 2651-62.

14. Hao L, Liao Q, Tang Q, Deng H, Chen L. Id-1 promotes osteosarcoma cell growth and inhibits cell apoptosis via PI3K/AKT signaling pathway. Biochemical and biophysical research communications. 2016; 470: 643-9.

15. Han SS, Han S, Kamberos NL. Piperlongumine inhibits the proliferation and survival of B-cell acute lymphoblastic leukemia cell lines irrespective of glucocorticoid resistance. Biochemical and biophysical research communications. 2014; 452: 669-75.

16. Kaushal K, Antao AM, Kim KS, Ramakrishna S. Deubiquitinating enzymes in cancer stem cells: functions and targeted inhibition for cancer therapy. Drug discovery today. 2018; 23: 1974-82.

17. Ogrunc M, Martinez-Zamudio RI, Sadoun PB, Dore G, Schwerer H, Pasero P, et al. USP1 Regulates Cellular Senescence by Controlling Genomic Integrity. Cell reports. 2016; 15: 1401-11.

18. Guervilly JH, Renaud E, Takata M, Rosselli F. USP1 deubiquitinase maintains phosphorylated CHK1 by limiting its DDB1-dependent degradation. Human molecular genetics. 2011; 20: 2171-81.

19. Sachdeva R, Wu M, Smiljanic S, Kaskun O, Ghannad-Zadeh K, Celebre A, et al. ID1 Is Critical for Tumorigenesis and Regulates Chemoresistance in Glioblastoma. Cancer research. 2019; 79: 4057-71.

20. Zhao GS, Zhang Q, Cao Y, Wang Y, Lv YF, Zhang ZS, et al. High expression of ID1 facilitates metastasis in human osteosarcoma by regulating the sensitivity of anoikis via PI3K/AKT depended suppression of the intrinsic apoptotic signaling pathway. American journal of translational research. 2019; 11: 2117-39.

21. Zhao Z, Bo Z, Gong W, Guo Y. Inhibitor of Differentiation 1 (Id1) in Cancer and Cancer Therapy. International Journal of Medical Sciences. 2020; 17: 995-1005.

22. Cruz-Rodriguez N, Combita AL, Enciso LJ, Quijano SM, Pinzon PL, Lozano OC, et al. High expression of ID family and IGJ genes signature as predictor of low induction treatment response and worst survival in adult Hispanic patients with B-acute lymphoblastic leukemia. Journal of experimental \& clinical cancer research : CR. 2016; 35: 64.

23. Wang L, Man N, Sun XJ, Tan Y, Garcia-Cao M, Liu F, et al. Regulation of AKT signaling by Id1 controls $t(8 ; 21)$ leukemia initiation and progression. Blood. 2015; 126: 640-50.

24. Alzahrani AS. PI3K/Akt/mTOR inhibitors in cancer: At the bench and bedside. Seminars in cancer biology. 2019; 59:125-32.

25. Curigliano G, Shah RR. Safety and Tolerability of Phosphatidylinositol-3-Kinase (PI3K) Inhibitors in Oncology. Drug safety. 2019; 42: 247-62

26. Gomes AM, Soares MV, Ribeiro P, Caldas J, Povoa V, Martins LR, et al. Adult B-cell acute lymphoblastic leukemia cells display decreased PTEN activity and constitutive hyperactivation of PI3K/Akt pathway despite high PTEN protein levels. Haematologica. 2014; 99: 1062-8.

27. Bayati S, Bashash D, Ahmadian S, Safaroghli-Azar A, Alimoghaddam K, Ghavamzadeh A, et al. Inhibition of tachykinin NK1 receptor using aprepitant induces apoptotic cell death and G1 arrest through Akt/p53 axis in pre-B acute lymphoblastic leukemia cells. European journal of pharmacology. 2016; 791: $274-83$. 